\title{
Asymmetrically protected porphyrin meso-tetraphenols and their application in the synthesis of pentaporphyrin dendrimers.
}

\author{
Stefaan Smeets, Hilde Roex and Wim Dehaen* \\ Department of Chemistry, Katholieke Universiteit Leuven, Celestijnenlaan 200F, \\ 3001 Leuven (Belgium) \\ E-mail :wim.dehaen@chem.kuleuven.ac.be
}

Dedicated to Professor Georges Hoornaert on his $65^{\text {th }}$ birthday

(received 16 Jan 03; accepted 07 Mar 03; published on the web 24 Mar 03)

\begin{abstract}
The readily available tetrakis(4-hydroxyphenyl)porphyrin core $\mathbf{1}$ was silylated to the trisprotected 2a and the tetraprotected 3a. These two molecules were used, respectively as monomers and core reagents in a synthesis of pentaporphyrin dendrimers 7a-c with benzyl ether branches or hexadecyl groups as the peripheral groups.
\end{abstract}

Keywords: Dendrimers, porphyrins, protection

\section{Introduction}

Numerous research groups have used porphyrins or metalloporphyrins as central building blocks (cores) in dendrimers. [1-11] The first example was described by Inoue and Aida. [1] It has been suggested that dendritic porphyrins could act as model sytems for natural electron transfer hemoproteins such as cytochrome c or hemoglobin. [2,3] Because of their large size and the possibility of host-guest interactions, the porphyrins represent attractive cores for the design of dendritic sensors and catalysts. Introduction of bulky dendrons at the peripheral positions of a metalloporphyrin results in steric protection and could provide regio- and stereoselective catalysis. [4]

In earlier work, we described benzyl ether dendrimers derived from the readily available tetrakis(4-hydroxyphenyl)porphyrin core 1. [11] We wanted to devise a synthesis of dendrimers having porphyrins at several levels of the macromolecule. [12] Therefore, it was necessary to desymmetrize 1. We previously have used the t-butyldiphenylsilyl (TBDPS) group previously $[13,14]$ as a protecting group for the 3,5-dihydroxybenzyl alcohol monomer in the accelerated synthesis of benzyl ether [15] dendrons previously described by Fréchet. An additional 
advantage of the bulky silyl group is its solubilizing ability, which adresses the main problem associated with working with $\mathbf{1}$.

\section{Results and Discussion}

The tetraphenol $\mathbf{1}$ is readily available in $95 \%$ yield from the corresponding tetramethoxy derivative by demethylation with pyridinium hydrochloride. Then, reaction of $\mathbf{1}$ with 4.4 equivalents of TBDPS-Cl and nine equivalents of imidazole gave a mixture of products from which the trisprotected $\mathbf{2 a}$ and the tetraprotected 3a could be isolated by flash chromatography, respectively in 34 and 38 \% yield. As expected, 2a and 3a had excellent solubilities in most organic solvents. In comparison, alkylation of $\mathbf{1}$ with 1-bromohexadecane was very sluggish and gave, after painstaking chromatography, the trisalkylated and tetraalkylated porphyrins $\mathbf{2 b}$ and 3b in 10 and $12 \%$ yield, respectively. The use of the G1 benzylic bromide to desymmetrize $\mathbf{1}$ afforded only $24 \%$ of the desired 2 c, together with $60 \%$ of the tetrasubstituted $3 c$. The products $\mathbf{2 b - c}$ and $\mathbf{3 b}$-c were much less soluble in organic solvents than their silylated analogs $\mathbf{2 a}$ and $3 \mathbf{a}$.

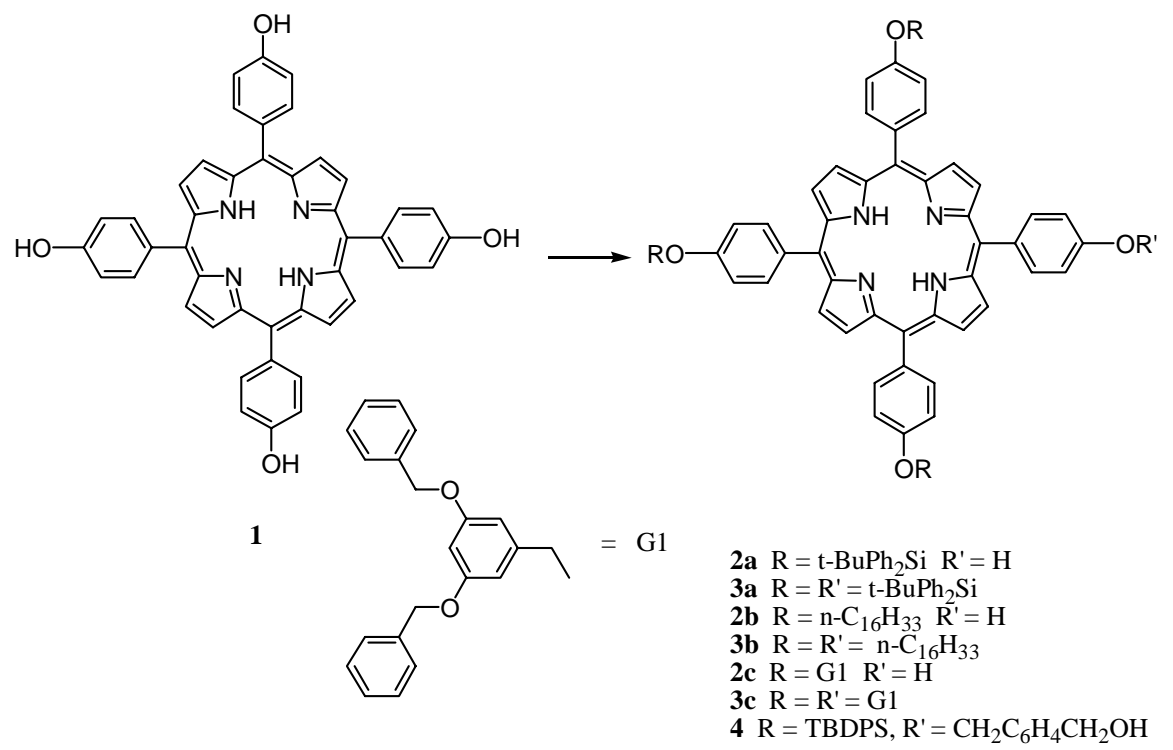

Mitsunobu reaction of 2a with an excess of 1,4-benzenedimethanol gave a good yield (85\%) of the porphyrin 4, having a single benzyl alcohol function. Reaction of $\mathbf{4}$ with the benzylic G1$\mathrm{Br}$, using deprotection/realkylation conditions described earlier $[13,14]$, gave the corresponding dendron 5 a in $95 \%$ yield. Bromination using soft conditions $\left(\mathrm{CBr}_{4} / \mathrm{PPh}_{3}\right)$ gave the bromide $\mathbf{6 a}$ in $83 \%$ yield. In preliminary work, 6a was obtained in $70 \%$ yield from 2c and 1,4bis(bromomethyl)benzene. The pentaporphyrin 7a was then prepared in $48 \%$ yield by combining the tetraprotected porphyrin core $\mathbf{3 a}$ with the bromide $\mathbf{6 a}$ under deprotection/realkylation conditions.

The same reaction sequence, starting from 4 and using 1-bromohexadecane instead of G1-Br, gave $90 \%$ of the dendron $\mathbf{5 b}, 65 \%$ of bromide $\mathbf{6 b}$ (90\% from $\mathbf{2 b}$ and 1,4-bis(bromomethyl)- 
benzene) and $18 \%$ of the pentaporphyrin $\mathbf{7 b}$. Starting from $\mathbf{4}$ and the Fréchet G2-Br, $\mathbf{5 c}$ (70 \%), 6c (80 \%) were obtained. The final pentaporphyrin 7c was prepared from $\mathbf{6 c}$ and $\mathrm{Zn}[\mathbf{1}]$ (not 3a) in $40 \%$ yield. Only a very small amount of $\mathrm{Zn}[\mathbf{1}]$ was necessary, so there were no solubility problems in this case. Thus, this modular approach allows the preparation of oligoporphyrins metallated at a chosen level within the dendrimer structure.

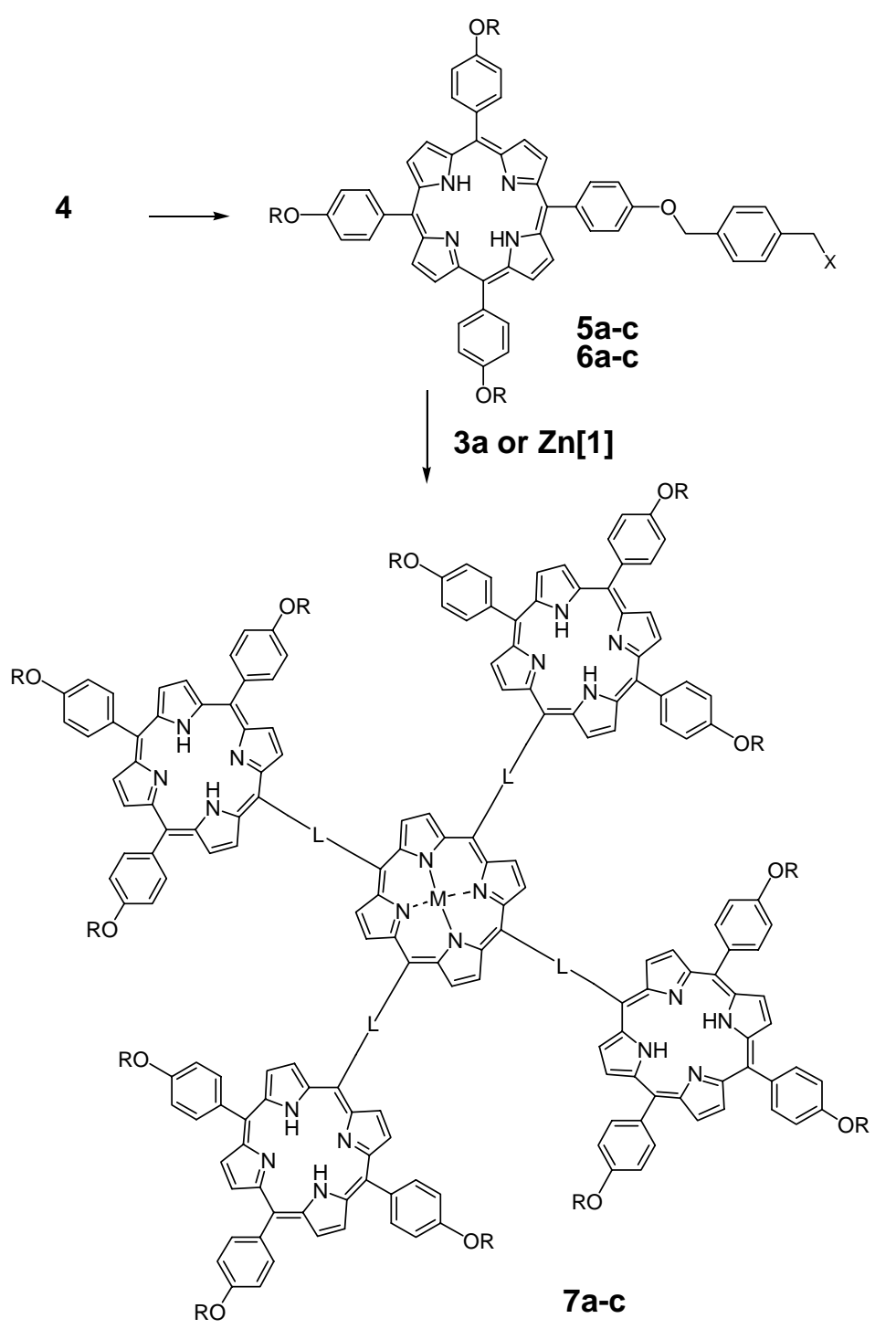




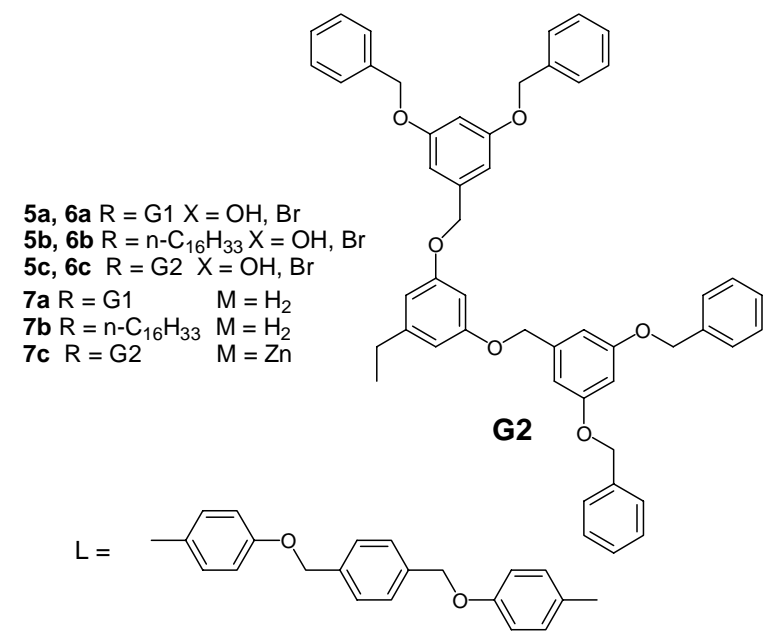

The pentaporphyrins 7a-c were characterized by ${ }^{1} \mathrm{H}$ NMR and ${ }^{13} \mathrm{C}$ NMR spectroscopy (for $\mathbf{7 a}$ and $\mathbf{7 b}$ ), MALDI-TOF mass spectrometry and GPC (for $\mathbf{7 a}$ and $\mathbf{7 c}$ ). The latter technique gave an $\mathrm{M}_{\mathrm{n}}$ (respectively 7513 and 11305) that was very close to the theoretically expected one (7496 and 12588), proving that these dendrimers still have a very open structure. Also, the dendrimers $7 \mathbf{a}$ and $\mathbf{7 c}$ are essentially monodisperse $(\mathrm{PD}=1.04)$. All dendrimers $\mathbf{7 a - c}$ gave only the theoretically expected MALDI TOF MS peak for $\mathrm{MH}^{+}$.

\section{Conclusions}

The tetrakis(4-hydroxyphenyl)porphyrin $\mathbf{1}$ can be desymmetrized to the trisilylated 2a and the latter compound can be used as a soluble building block for the synthesis of pentaporphyrin systems 7a-c, possessing Fréchet dendrons (first and second generation) or hexadecyl groups as the terminal groups.

\section{Experimental Section}

General Procedures. ${ }^{1} \mathrm{H}$ NMR or ${ }^{13} \mathrm{C}$ NMR spectra were measured with a $400 \mathrm{MHz}$ Bruker apparatus. Mass spectra were obtained on a Micromass Quatro II in ESI (infusion $50 \mu \mathrm{l}$ $\mathrm{MeOH} / \mathrm{CH}_{2} \mathrm{Cl}_{2}-\mathrm{NH}_{4} \mathrm{OAc}(0.1 \mathrm{M}$ in $\mathrm{MeOH}$ ) with a Harvard pump, model 11) or in APCI (250 $\mu \mathrm{l}$ $\mathrm{MeOH} / \mathrm{CH}_{2} \mathrm{Cl}_{2}$ using a Hewlett Packard HP 1100 binary pump and infusion of 20-30 $\mu \mathrm{l}$ $\mathrm{MeOH} / \mathrm{CH}_{2} \mathrm{Cl}_{2}-\mathrm{NH}_{4} \mathrm{OAc}(0.1 \mathrm{M}$ in $\mathrm{MeOH}$ ) with a Harvard pump model 11). The higher molecular mass compounds were measured with a MALDI-TOF apparatus, using a tetrahydrofuran solution of indoleacrylic acid $(0.4 \mathrm{M})$ as the matrix. GPC (Waters apparatus) used polystyrene standards and absorbance detection. 
5,10,15,20-Tetrakis(4-hydroxyphenyl)porphyrin (1). A mixture of pyridine (64 ml) and hydrochloric acid $(72 \mathrm{ml})$ was evaporated during $1 \mathrm{~h}$ until an inner temperature of at least $170^{\circ} \mathrm{C}$ was reached. Then the tetrakis(4-methoxyphenyl)porphyrin (10 g) was added. The mixture was heated at reflux while stirring for a further $3 \mathrm{~h}$. After cooling, water $(200 \mathrm{ml})$ was added and the resulting precipitate was filtered and washed with a saturated sodium acetate solution. The resulting solid was redissolved in a mixture of acetone $(150 \mathrm{ml})$ and triethylamine $(5 \mathrm{ml})$. Then, $600 \mathrm{ml}$ of hexane was added. The resulting precipitate (95\% yield) was isolated and consists of 1, of sufficient purity for further synthesis.

${ }^{1} \mathrm{H}$ NMR (400 MHz, $\mathrm{CDCl}_{3}$ ) $\delta 9.94$ (s, 4H, OH), 8.88 (s, 8H, $\beta$-pyrrole), 8.01 (d, $J=7.6 \mathrm{~Hz}, 8 \mathrm{H}$, 2,6-phenyl-H), 7.22 (d, $J=7.6 \mathrm{~Hz}, 8 \mathrm{H}, 3,5-$ phenyl-H), -2.83 (s, 2H, NH) ; ${ }^{13} \mathrm{C}$ NMR (100 MHz, $\left.\mathrm{CDCl}_{3}\right) \delta 157.4,135.5,131.9,131.3,120.0,113.9$.

Zn[1] : A solution of $1(0.50 \mathrm{~g}, 0.73 \mathrm{mmol})$ in methanol $(20 \mathrm{ml})$ was treated with zinc acetate (1.35g, $7.40 \mathrm{mmol})$. The mixture was stirred for $2 \mathrm{~h}$ at room temperature, poured in ethyl acetate $(50 \mathrm{ml})$ and washed with water (3x $50 \mathrm{ml})$. After drying on magnesium sulfate, the solvent was removed in vacuo and the residue chromatographed over silica with a ethyl acetate / hexane 3:2 mixture. This gave Zn [1](0.49g, $90 \%)$.

${ }^{1} \mathrm{H}$ NMR (400 MHz, $\mathrm{CDCl}_{3}$ ) $\delta 9.80$ (s, 4H, OH), 8.80 (s, 8H, $\beta$-pyrrole), 7.94 (d, $J=8.3 \mathrm{~Hz}, 8 \mathrm{H}$, 2,6-phenyl-H), 7.16 (d, $J=8.3 \mathrm{~Hz}, 8 \mathrm{H}, 3,5$-phenyl-H); ${ }^{13} \mathrm{C} \mathrm{NMR}\left(100 \mathrm{MHz}, \mathrm{CDCl}_{3}\right) \delta 156.8$, 149.6, 135.2, 133.4, 131.3, 120.2, 113.5; UV/Vis ( $\lambda_{\max }, \mathrm{nm}$, dichloromethane) 424.0, 557.1, 598.2; ESI MS $742\left(\mathrm{MH}^{+}\right)$.

Silylation of 1. Porphyrin 1 (2.00 g, $2.90 \mathrm{mmol}$ ), chloro-t-butyldiphenylsilane (3.57 g, $13 \mathrm{mmol}$ ) and imidazole (1.77 g, $26 \mathrm{mmol})$ were dissolved in dimethylformamide (30 ml) and stirred at ambient temperature under inert atmosphere for $12 \mathrm{~h}$. The solvent was removed in vacuo and the residue chromatographed over silica with a dichloromethane/hexane 1:1 mixture. This gave 3a (1.81g, $38 \%)$ as a first fraction. Further elution gave 2a (1.38g, $34 \%)$.

5-(4-Hydroxyphenyl)-10,15,20-tris(4-t-butyldiphenylsilyloxyphenyl)porphyrin (2a). ${ }^{1} \mathrm{H}$ NMR $\left(400 \mathrm{MHz}, \mathrm{CDCl}_{3}\right) \delta 8.82$ (s, 4H, $\beta$-pyrrole), 8.79 (d, $J=4.8 \mathrm{~Hz}, 2 \mathrm{H}, \beta$-pyrrole), 8.73 (d, $J=$ $4.8 \mathrm{~Hz}, 2 \mathrm{H}, \beta$-pyrrole), 8.01 (d, $J=8.4 \mathrm{~Hz}, 2 \mathrm{H}), 7.93$ (m, 12H), 7.86 (d, $J=9.0 \mathrm{~Hz}, 6 \mathrm{H}), 7.53-$ 7.46 (m, 18H), 7.14 (d, $J=8.4 \mathrm{~Hz}, 2 \mathrm{H}), 7.13$ (d, $J=9.0 \mathrm{~Hz}, 6 \mathrm{H}), 1.22$ (s, $27 \mathrm{H}, \mathrm{t}-\mathrm{Bu}$ ), -2.90 (s, $2 \mathrm{H}, \mathrm{NH}) ; \quad{ }^{13} \mathrm{C}$ NMR $\left(100 \mathrm{MHz}, \mathrm{CDCl}_{3}\right) \delta 155.6,155.3,135.8,135.7,135.3,135.1,134.9$, 133.1, 130.1, 127.9, 119.8, 119.4, 118.2, 113.6, 26.7, 19.7; UV/Vis ( $\lambda_{\max }, \mathrm{nm}$, dichloromethane) 421.1, 518.3, 555.0, 593.4, 649.9; ESI MS $1395\left(\mathrm{MH}^{+}\right)$.

5,10,15,20-Tetrakis(4-t-butyldiphenylsilyloxyphenyl)porphyrin (3a). ${ }^{1} \mathrm{H}$ NMR (400 $\mathrm{MHz}$, $\left.\mathrm{CDCl}_{3}\right) \delta 8.70$ (s, 8H, $\beta$-pyrrole), 7.93 (m, 16H), 7.86 (d, $\left.J=8.4 \mathrm{~Hz}, 8 \mathrm{H}\right), 7.51$ (m, 24H), 7.12 $(\mathrm{d}, J=8.4 \mathrm{~Hz}, 8 \mathrm{H}), 1.20$ (s, 36H, t-Bu), -2.90 (s, 2H, NH); ${ }^{13} \mathrm{C}$ NMR $\left(100 \mathrm{MHz}, \mathrm{CDCl}_{3}\right) \delta$ 155.6, 135.8, 135.3, 135.1, 133.1, 130.1, 127.9, 119.7, 118.2, 26.7, 19.7; UV/Vis $\left(\lambda_{\max }, \mathrm{nm}\right.$, dichloromethane) 421.4, 518.7, 555.4, 593.9, 649.6; ESI MS $1633\left(\mathrm{MH}^{+}\right)$.

Hexadecylation of 1. Porphyrin 1 (1g, $1.47 \mathrm{mmol})$, 1-bromohexadecane (1.49 g, $4.87 \mathrm{mmol}$ ) and potassium carbonate $(0.67 \mathrm{~g}, 4.85 \mathrm{mmol})$ were added to $30 \mathrm{ml}$ of dimethylformamide. The mixture was heated at reflux under argon for $48 \mathrm{~h}$. After cooling, $50 \mathrm{ml}$ of dichloromethane was 
added and the organic layer was washed with water (3x50ml), and dried on magnesium sulfate. The solvent was removed in vacuo and the residue chromatographed over silica with a dichloromethane / hexane 1:1 mixture. This gave the known [16] 3b $(0.21 \mathrm{~g}, 12 \%)$ as a first fraction. Further elution gave $\mathbf{2 b}(0.24 \mathrm{~g}, 10 \%)$.

5-(4-Hydroxyphenyl)-10,15,20-tris(4-n-hexadecyloxyphenyl)porphyrin (2b). ${ }^{1} \mathrm{H}$ NMR (400 MHz, $\mathrm{CDCl}_{3}$ ) $\delta 8.85$ (s, 4H, $\beta$-pyrrole), 8.82 (d, $J=4.8 \mathrm{~Hz}, 2 \mathrm{H}, \beta$-pyrrole), 8.80 (d, $J=4.8 \mathrm{~Hz}$, 2H, $\beta$-pyrrole), 8.07 (d, $J=8.1 \mathrm{~Hz}, 6 \mathrm{H}$ ), 8.01 (d, $J=8.0 \mathrm{~Hz}, 2 \mathrm{H}$ ), 7.23 (d, $J=8.1 \mathrm{~Hz}, 6 \mathrm{H}$ ), 7.13 (d, $J=8.0 \mathrm{~Hz}, 2 \mathrm{H}), 4.17$ (t, $J=6.4 \mathrm{~Hz}, 6 \mathrm{H}), 1.94(\mathrm{~m}, 6 \mathrm{H}), 1.59$ (m, 6H), 1.27 (m, $72 \mathrm{H}), 0.88$ (t, $J=7.0 \mathrm{~Hz}, 9 \mathrm{H}),-2.70(\mathrm{~s}, 2 \mathrm{H}, \mathrm{NH}) ;{ }^{13} \mathrm{C} \mathrm{NMR}\left(100 \mathrm{MHz}, \mathrm{CDCl}_{3}\right) \delta$ 159.0, 155.2, 135.6, 135.5, 135.4, 130.9, 119.9, 113.5, 112.7, 68.3, 31.9, 29.7, 29.5, 29.4, 26.9, 26.5, 22.7, 14.1; UV/Vis ( $\lambda_{\max }, \mathrm{nm}$, dichloromethane) 421.7, 517.9, 555.8, 594.4, 649.9; ESI MS $1351\left(\mathrm{MH}^{+}\right)$.

Dendronisation (G1) of 1. A mixture of porphyrin 1 (1g, $1.47 \mathrm{mmol})$, G1-Br (1.86g, 4.87 mmol), potassium carbonate $(1.34 \mathrm{~g}, 9.69 \mathrm{mmol})$ and 18 -crown-6 $(40 \mathrm{mg}, 0.15 \mathrm{mmol})$ in dimethylformamide ( $30 \mathrm{ml}$ ) was heated while stirring in argon at $80^{\circ} \mathrm{C}$ for $48 \mathrm{~h}$. After removal of the solvent in vacuo, the residue was dissolved in dichloromethane $(50 \mathrm{ml})$ and washed with water ( $3 \times 50 \mathrm{ml})$. The organic layer was dried on magnesium sulfate. The solvent was removed in vacuo and the residue chromatographed over silica with a dichloromethane / hexane 9:1 mixture. This gave the known [11] 3c (1.67g, $60 \%)$ as a first fraction. Further elution gave 2c (0.56g, 24 $\%)$.

5-(4-Hydroxyphenyl)-10,15,20-tris(4-(3,5-bis(benzyloxy)benzyloxy)phenyl)porphyrin (2c). ${ }^{1} \mathrm{H}$ NMR (400 MHz, $\mathrm{CDCl}_{3}$ ) $\delta 8.85$ (s, 8H, $\beta$-pyrrole), 8.09 (d, $J=8.5 \mathrm{~Hz}, 6 \mathrm{H}$ ), 8.05 (d, $J=8.4$ $\mathrm{Hz}, 2 \mathrm{H}), 7.47$ (d, $J=7.9 \mathrm{~Hz}, 12 \mathrm{H}), 7.38(\mathrm{~m}, 12 \mathrm{H}), 7.32$ (d, $J=8.5 \mathrm{~Hz}, 6 \mathrm{H}), 7.30$ (m, 6H), 7.16 (d, $J=8.5 \mathrm{~Hz}, 6 \mathrm{H}$ ), 6.79 (d, $J=2.0 \mathrm{~Hz}, 6 \mathrm{H}), 6.66$ (t, $J=2.0 \mathrm{~Hz}, 3 \mathrm{H}$ ), 5.26 (s, 6H), 5.13 (s, $12 \mathrm{H}$ ), $-2.70(\mathrm{~s}, 2 \mathrm{H}, \mathrm{NH}) ;{ }^{13} \mathrm{C}$ NMR $\left(100 \mathrm{MHz}, \mathrm{CDCl}_{3}\right) \delta 160.3,158.6,155.2,139.5,136.9,135.7(2)$, 135.0, 134.9, 128.6, 128.0, 127.6, 119.7, 113.7, 113.2, 113.0, 106.7, 101.8, 70.3, 67.1; UV/Vis ( $\lambda_{\max }, \mathrm{nm}$, dichloromethane) 421.6, 518.7, 555.3, 593.2, 650.3; ESI MS $1589\left(\mathrm{MH}^{+}\right)$.

5,10,15-Tris(4-t-butyldiphenylsilyloxyphenyl)-20-(4-(4-(hydroxymethyl)benzyloxy)phenyl)porphyrin (4). Porphyrin 2a (0.50g, 0.36mmol), 1,4-benzenedimethanol (0.49g, 3.59 mmol), diethyl azodicarboxylate $(90 \mathrm{mg}, 0.54 \mathrm{mmol})$ and triphenylphosphine $(0.14 \mathrm{~g}, 0.54$ mmol) were dissolved in dry tetrahydrofuran $(5 \mathrm{ml})$. The mixture was stirred for $3 \mathrm{~h}$ at ambient temperature, evaporated in vacuo and redissolved in dichloromethane $(15 \mathrm{ml})$. After washing with water $(3 \times 20 \mathrm{ml})$ the organic layer was dried over magnesium sulfate. The solvent was removed in vacuo and the residue chromatographed over silica with a dichloromethane / hexane 3:1 mixture. This afforded the porphyrin $4(0.47 \mathrm{~g}, 85 \%)$.

${ }^{1} \mathrm{H}$ NMR (400 MHz, $\left.\mathrm{CDCl}_{3}\right) \delta 8.80$ (d, $J=4.7 \mathrm{~Hz}, 2 \mathrm{H}, \beta$-pyrrole), 8.73 (d, $J=4.7 \mathrm{~Hz}, 2 \mathrm{H}, \beta$ pyrrole), 8.72 (s, 4H, $\beta$-pyrrole), 8.08 (d, $J=8.6 \mathrm{~Hz}, 2 \mathrm{H}$ ), 7.93 (m, $12 \mathrm{H}$ ), 7.87 (d, $J=8.4 \mathrm{~Hz}$, $6 \mathrm{H}), 7.61$ (d, $J=7.9 \mathrm{~Hz}, 2 \mathrm{H}), 7.55-7.45(\mathrm{~m}, 18 \mathrm{H}), 7.49$ (d, $J=5.1 \mathrm{~Hz}, 2 \mathrm{H}), 7.33(\mathrm{~d}, J=8.6 \mathrm{~Hz}$, 2H), 7.12 (d, $J=8.4 \mathrm{~Hz}, 6 \mathrm{H}), 5.34$ (s, 2H), 4.77 (d, $J=5.6 \mathrm{~Hz}, 2 \mathrm{H}), 1.49$ (t, $J=5.6 \mathrm{~Hz}, 1 \mathrm{H}$ ), 1.28 (s, 27H), -2.90 (s, 2H, NH); ${ }^{13} \mathrm{C}$ NMR $\left(100 \mathrm{MHz}, \mathrm{CDCl}_{3}\right) \delta$ 158.6, 155.6, 140.9, 136.6, 135.8, 135.6, 135.3, 135.1, 135.0, 133.1, 131.0, 128.0, 127.9, 127.3, 119.8, 119.5, 118.2, 113.1, 
70.1, 65.2, 26.8, 19.7; UV/Vis ( $\lambda_{\max }$, nm, dichloromethane) 422.4, 518.4, 555.5, 593.5, 649.5; ESI MS $1516\left(\mathrm{MH}^{+}\right)$.

\section{General procedure for deprotection/realkylation}

5,10,15-Tris(4-(3,5-bis(benzyloxy)-benzyloxy)phenyl)-20-(4-(4-(hydroxymethyl)benzyloxy) phenyl)porphyrin (5a). Porphyrin 4 (180 mg, $0.12 \mathrm{mmol}), \mathrm{G} 1-\mathrm{Br}$ (0.21g, $0.55 \mathrm{mmol})$, potassium fluoride (58 mg, $1 \mathrm{mmol})$ and 18-crown-6 $(0.26 \mathrm{~g}, 0.98 \mathrm{mmol})$ were added to acetone $(10 \mathrm{ml})$ and heated at reflux for $24 \mathrm{~h}$ while stirring under an argon atmosphere. After cooling, the mixture was added to dichloromethane $(25 \mathrm{ml})$ and the organic layer was washed with water (3x30ml) and dried over magnesium sulfate. The solvent was removed in vacuo and the residue chromatographed over silica with a dichloromethane / diethyl ether 19:1 mixture. This afforded the porphyrin 5a (0.17g, $90 \%) .{ }^{1} \mathrm{H}$ NMR (400 MHz, $\left.\mathrm{CDCl}_{3}\right) \delta 8.08$ (s, 8H, $\beta$-pyrrole), 8.06 (d, $J$ $=8.6 \mathrm{~Hz}, 6 \mathrm{H}), 7.53(\mathrm{~d}, J=8.0 \mathrm{~Hz}, 2 \mathrm{H}), 7.45(\mathrm{~m}, 12 \mathrm{H}), 7.40$ (d, $J=8.0 \mathrm{~Hz}, 2 \mathrm{H}), 7.38(\mathrm{~m}, 12 \mathrm{H})$, 7.30 (m, 6H), 7.24 (d, $J=8.6 \mathrm{~Hz}, 6 \mathrm{H}), 6.84$ (d, $J=2.2 \mathrm{~Hz}, 6 \mathrm{H}), 6.65$ (t, $J=2.2 \mathrm{~Hz}, 3 \mathrm{H}), 5.22$ (s, 2H), 5.17 (s, 2H), 5.16 (s, 4H), 5.10 (s, 12H), 4.68 (s, 2H), 1.64 (s, 1H), -2.30 (s, $2 \mathrm{H}, \mathrm{NH}) ;{ }^{13} \mathrm{C}$ NMR (100 MHz, $\left.\mathrm{CDCl}_{3}\right) \delta$ 160.3, 158.6, 158.5, 140.8, 139.4, 136.9, 136.4, 135.6, 135.4, 134.9, 131.0, 128.6, 128.0, 127.8, 127.6, 127.2, 119.7, 113.1, 70.2, 70.0; UV/Vis ( $\lambda_{\max }, \mathrm{nm}$, dichloromethane) 421.4, 518.5, 555.8, 593.5, 650.1; ESI MS $1708\left(\mathrm{MH}^{+}\right)$.

\section{5,10,15-Tris(4-n-hexadecyloxyphenyl)-20-(4-(4-(hydroxymethyl)benzyloxy)-}

phenyl)porphyrin (5b). Porphyrin 5b was prepared analogously in $90 \%$ yield from 4 and 1bromohexadecane. The reaction was carried out at room temperature in dimethylformamide (24h). ${ }^{1} \mathrm{H}$ NMR (400 MHz, $\mathrm{CDCl}_{3}$ ) $\delta 8.85$ (s, 4H, $\beta$-pyrrole), 8.84 (d, $J=4.7 \mathrm{~Hz}, 2 \mathrm{H}, \beta$-pyrrole), 8.83 (d, $J=4.7 \mathrm{~Hz}, 2 \mathrm{H}, \beta$-pyrrole), 8.07 (d, $J=8.4 \mathrm{~Hz}, 8 \mathrm{H}), 7.53$ (d, $J=8.0 \mathrm{~Hz}, 2 \mathrm{H}), 7.41$ (d, $J$ $=8.0 \mathrm{~Hz}, 2 \mathrm{H}), 7.26(\mathrm{~d}, J=8.4 \mathrm{~Hz}, 2 \mathrm{H}), 7.22(\mathrm{~d}, J=8.4 \mathrm{~Hz}, 6 \mathrm{H}), 5.22(\mathrm{~s}, 2 \mathrm{H}), 4.70(\mathrm{~s}, 2 \mathrm{H}), 4.18$ (t, $J=6.4 \mathrm{~Hz}, 6 \mathrm{H}), 3.60$ (s, 1H), 1.94 (s, 6H), 1.58 (s, 6H), 1.27 (m, 72H), 0.86 (t, J = 7.0 Hz, 9H), -2.70 (s, 2H, NH); ${ }^{13} \mathrm{C}$ NMR (100 MHz, $\left.\mathrm{CDCl}_{3}\right) \delta 159.0,158.6,140.9,136.5,135.6,135.0$, 134.4, 127.9, 127.2, 119.8, 119.5, 113.1, 112.7, 70.1, 68.3, 65.1, 31.9, 29.6, 29.5, 29.4, 26.2, 25.7, 22.7, 14.1; UV/Vis ( $\lambda_{\max }$, nm, dichloromethane) 422.1, 518.9, 556.2, 595.0, 650.8; ESI MS $1473\left(\mathrm{MH}^{+}\right)$.

5,10,15-Tris(4-(3,5-bis(3,5-bis(benzyloxy)benzyloxy)benzyloxy)phenyl)-20-(4-(4(hydroxymethyl)benzyloxy)phenyl)porphyrin (5c). Porphyrin 5c was prepared analogously in $70 \%$ yield from 4 and G2-Br. ${ }^{1} \mathrm{H}$ NMR $\left(400 \mathrm{MHz}, \mathrm{CDCl}_{3}\right) \quad \delta 8.85(\mathrm{~s}, 8 \mathrm{H}, \beta-$ pyrrole), 8.08 (d, $J=8.5 \mathrm{~Hz}, 8 \mathrm{H}), 7.57$ (d, $J=7.8 \mathrm{~Hz}, 2 \mathrm{H}), 7.46$ (d, $J=7.8 \mathrm{~Hz}, 2 \mathrm{H}$ ), 7.45-7.25 $(\mathrm{m}, 68 \mathrm{H}), 6.85(\mathrm{~d}, J=2.3 \mathrm{~Hz}, 6 \mathrm{H}), 6.73(\mathrm{~d}, J=2.3 \mathrm{~Hz}, 12 \mathrm{H}), 6.63$ (t, $J=2.3 \mathrm{~Hz}, 3 \mathrm{H}), 6.58$ (t, $J$ $=2.3 \mathrm{~Hz}, 6 \mathrm{H}), 5.28(\mathrm{~s}, 2 \mathrm{H}), 5.21(\mathrm{~s}, 6 \mathrm{H}), 5.04(\mathrm{~s}, 12 \mathrm{H}), 5.02(\mathrm{~s}, 24 \mathrm{H}), 4.72(\mathrm{~d}, J=4.2 \mathrm{~Hz}, 2 \mathrm{H})$, 1.71 (t, $J=4.2 \mathrm{~Hz}, 1 \mathrm{H}),-2.75(\mathrm{~s}, 2 \mathrm{H}, \mathrm{NH}) ;{ }^{13} \mathrm{C}$ NMR $\left(100 \mathrm{MHz}, \mathrm{CDCl}_{3}\right) \delta 160.2,158.6,140.8$, 139.5, 139.3, 136.7, 135.6, 135.0, 128.5, 127.9, 127.5, 127.3, 119.7, 113.1, 106.7, 106.5, 101.8, 101.6, 70.3, 70.1, 65.1; MALDI TOF $2980.5\left(\mathrm{MH}^{+}\right)$.

\section{General procedure for bromination}

5,10,15-Tris(4-(3,5-bis(benzyloxy)benzyloxy)-phenyl)-20-(4-(4-(bromomethyl)benzyloxy) phenyl)porphyrin (6a). To a solution of porphyrin 5a $(0.50 \mathrm{~g}, 0.29 \mathrm{mmol})$ and $\mathrm{CBr}_{4}(0.292 \mathrm{~g}$, 
$0.88 \mathrm{mmol})$ in dry tetrahydrofuran $(5 \mathrm{ml})$ was added triphenylphosphine $(0.231 \mathrm{~g}, 0.88 \mathrm{mmol})$ and the mixture was stirred for $1 \mathrm{~h}$ at ambient temperature and then added to water $(20 \mathrm{ml})$. The mixture was extracted $(3 \times 20 \mathrm{ml})$ with dichloromethane and the organic layer was dried over magnesium sulfate. The solvent was removed in vacuo and the residue chromatographed over silica with a dichloromethane / hexane 3:1 mixture. This yielded 6a (0.43g, 83 \%). Alternatively, 6a was obtained in $70 \%$ yield from 2c and 10 equivalents of 1,4-bis(bromomethyl)benzene using potassium carbonate (2 equivalents), 18-crown-6 (0.5 equivalents), in tetrahydrofuran at reflux for $12 \mathrm{~h} .{ }^{1} \mathrm{H} \mathrm{NMR}\left(400 \mathrm{MHz}, \mathrm{CDCl}_{3}\right) \delta 8.84$ (s, 8H, $\beta$-pyrrole), 8.08 (d, $J=8.05 \mathrm{~Hz}, 8 \mathrm{H}$ ), 7.55 (d, $J=7.8 \mathrm{~Hz}, 2 \mathrm{H}), 7.48$ (d, $J=7.8 \mathrm{~Hz}, 2 \mathrm{H}), 7.47$ (m, 12H), 7.39 (m, 6H), 7.30 (m, 12H), 7.29 (d, $J=8.3 \mathrm{~Hz}, 8 \mathrm{H}), 6.86$ (d, $J=2.0 \mathrm{~Hz}, 6 \mathrm{H}), 6.66$ (t, $J=2.0 \mathrm{~Hz}, 3 \mathrm{H}), 5.27$ (s, $2 \mathrm{H}), 5.23$ (s, 6H), 5.11 (s, 12H), 4.54 (s, 2H), -2.54 (s, 2H, NH); $\left.{ }^{13} \mathrm{C} \mathrm{NMR} \mathrm{(100} \mathrm{MHz,} \mathrm{CDCl}_{3}\right) \delta$ 160.3, 158.5, 158.2, 148.0, 139.5, 137.7, 137.4, 135.6, 135.0, 131.0, 129.4, 128.6, 128.0, 127.6, 119.6, 113.1; 106.6, 101.8, 70.2, 69.8, 69.7, 33.2; UV/Vis ( $\lambda_{\max }, \mathrm{nm}$, dichloromethane) 422.0, 518.6, 555.7, 593.7, 650.3; ESI MS $1769\left(\mathrm{MH}^{+}\right)$.

5,10,15-Tris(4-n-hexadecyloxyphenyl)-20-(4-(4-bromomethyl)benzyloxy)phenyl)-porphyrin

(6b). Porphyrin 6b was prepared analogously from $5 \mathrm{~b}$ in $65 \%$ yield. Alternatively, $6 \mathrm{~b}$ was obtained in $90 \%$ yield from $2 \mathrm{~b}$ and 10 equivalents of 1,4-bis(bromomethyl)benzene. ${ }^{1} \mathrm{H}$ NMR (400 MHz, $\mathrm{CDCl}_{3}$ ) $\delta 8.85$ (s, 8H, $\beta$-pyrrole), 8.83 (d, $J=4.6 \mathrm{~Hz}, 2 \mathrm{H}, \beta$-pyrrole), 8.81 (d, $J=4.6$ Hz, 2H, $\beta$-pyrrole), 8.08 (d, $J=8.0 \mathrm{~Hz}, 6 \mathrm{H}$ ), 8.05 (d, $J=7.7 \mathrm{~Hz}, 2 \mathrm{H}), 7.49$ (d, $J=8.0 \mathrm{~Hz}, 2 \mathrm{H}$ ), 7.44 (d, $J=8.0 \mathrm{~Hz}, 2 \mathrm{H}), 7.22$ (d, $J=7.7 \mathrm{~Hz}, 2 \mathrm{H}), 7.21$ (d, $J=8.0 \mathrm{~Hz}, 6 \mathrm{H}), 5.18$ (s, 2H), 4.52 (s, 2H), 4.17 (t, $J=6.4 \mathrm{~Hz}, 6 \mathrm{H}), 1.94(\mathrm{~m}, 6 \mathrm{H}), 1.59(\mathrm{~m}, 6 \mathrm{H}), 1.27(\mathrm{~m}, 72 \mathrm{H}), 0.88$ (t, $J=7.0 \mathrm{~Hz}, 9 \mathrm{H})$, -2.70 (s, 2H, NH); ${ }^{13} \mathrm{C}$ NMR (100 $\left.\mathrm{MHz} \mathrm{CDCl}_{3}\right) \delta 159.0,158.5,137.6,137.4,135.6,135.4$, $135.1,130.9,129.4,128.0,119.9,119.5,113.0,112.7,69.8,14.1 ;$ UV/Vis $\left(\lambda_{\max }, \mathrm{nm}\right.$, dichloromethane) 421.9, 518.7, 556.1, 593.9, 650.6; ESI MS $1533\left(\mathrm{MH}^{+}\right)$.

5,10,15-Tris(4-(3,5-bis(3,5-bis(benzyloxy)benzyloxy)benzyloxy)phenyl)-20-(4-(4(bromomethyl)benzyloxy)phenyl)porphyrin (6c). Porphyrin 6c was prepared analogously from $5 \mathrm{c}$ in $80 \%$ yield. ${ }^{1} \mathrm{H}$ NMR (400 $\left.\mathrm{MHz} \mathrm{CDCl}_{3}\right) \quad \delta 8.85$ (s, 8H, $\beta$-pyrrole), 8.08 (d, $J=8.4$ $\mathrm{Hz}, 8 \mathrm{H}), 7.55$ (d, $J=8.6 \mathrm{~Hz}, 2 \mathrm{H}), 7.48$ (d, $J=8.6 \mathrm{~Hz}, 2 \mathrm{H}), 7.45-7.24$ (m, $68 \mathrm{H}), 6.85$ (d, $J=2.0$ $\mathrm{Hz}, 6 \mathrm{H}), 6.73$ (d, $J=2.0 \mathrm{~Hz}, 12 \mathrm{H}), 6.63(\mathrm{t}, J=2.0 \mathrm{~Hz}, 3 \mathrm{H}), 6.58(\mathrm{t}, J=2.0 \mathrm{~Hz}, 6 \mathrm{H}), 5.27$ (s, 2H), 5.21 (s, 6H), 5.04 (s, 12H), 5.02 (s, 24H), 4.53 (s, 2H), -2.75 (s, 2H, NH) ; ${ }^{13} \mathrm{C}$ NMR (100 $\left.\mathrm{MHz} \mathrm{CDCl}_{3}\right) \delta 160.2,158.6,139.4,139.3,137.6,137.4,136.7,135.6,135.0,129.4,128.5$, 128.0, 127.5, 119.7, 113.1, 106.7, 106.4, 101.7, 101.6, 70.3, 70.1; MALDI TOF 3043.4 $\left(\mathrm{MH}^{+}\right)$.

\section{General procedure for synthesis of pentaporphyrins}

5, 10, 15, 20-Tetrakis(4-(4-(4-(5,10,15-tris(4-(3,5-bis(benzyloxy)benzyloxy)phenyl)porphyrin20-yl) fenoxymethyl)-benzyloxy)phenyl)porphyrin (7a). A solution of porphyrin 6a (0.24g, 0.13 mmol), porphyrin 3a (50 mg, $30 \mu \mathrm{mol})$, and 18-crown-6 (0.110 g, $0.42 \mathrm{mmol})$ in acetone (3ml) was treated with potassium fluoride (30 mg, $0.46 \mathrm{mmol}$ ). The resulting mixture was heated for $48 \mathrm{~h}$ at reflux under an argon atmosfere. After cooling, the mixture was added to dichloromethane $(25 \mathrm{ml})$ and the organic layer was washed with water $(3 \times 30 \mathrm{ml})$ and dried over magnesium sulfate. The solvent was removed in vacuo and the residue chromatographed over silica with a 
dichloromethane/hexane 3:1 mixture. The pentaporphyrin was further purified by precipitation from a dichloromethane solution in methanol to afford pure 7a $(0.11 \mathrm{~g}, 48 \%) .{ }^{1} \mathrm{H} \mathrm{NMR}(400 \mathrm{MHz}$, $\left.\mathrm{CDCl}_{3}\right) \quad \delta 8.84$ (s, 8H, $\beta$-pyrrole), 8.83 (m, 32H, $\beta$-pyrrole), 8.09 (m, 40H), 7.62 (s, 16H), 7.45$7.19(\mathrm{~m}, 160 \mathrm{H}), 6.81(\mathrm{~d}, J=2.0 \mathrm{~Hz}, 24 \mathrm{H}), 6.62$ (t, $J=2.0 \mathrm{~Hz}, 12 \mathrm{H}), 5.25$ (s, 8H), $5.21(\mathrm{~s}, 8 \mathrm{H})$, 5.14 (s, 8H), 5.12 (s, 16H), 5.08 (s, 16H), 5.06 (s, 32H), -2.70 (s, 10H, NH); UV/Vis ( $\lambda_{\max }, \mathrm{nm}$, dichloromethane) 422.6, 518.8, 556.0, 593.8, 650.4; $\mathrm{GPC}_{\mathrm{n}}=$ 7513, PD = 1.04; MALDI TOF $7496\left(\mathrm{MH}^{+}\right)$.

5, 10, 15, 20-Tetrakis(4-(4-(4-(5,10,15-tris(4-(n-hexadecyloxy)phenyl)-porphyrin-20-yl)fenoxymethyl)benzyloxy)phenyl)porphyrin (7b). Pentaporphyrin $7 \mathrm{~b}$ was prepared from $6 \mathrm{~b}$ and 4 in $18 \%$ yield. ${ }^{1} \mathrm{H}$ NMR (400 $\left.\mathrm{MHz} \mathrm{CDCl}_{3}\right) \quad \delta 8.85(\mathrm{~m}, 40 \mathrm{H}, \beta$-pyrrole), 8.11 (d, $J=8.6$ $\mathrm{Hz}, 40 \mathrm{H}$ ), 7.60 (d, $J=8.1 \mathrm{~Hz}, 8 \mathrm{H}), 7.47$ (d, $J=8.1 \mathrm{~Hz}, 8 \mathrm{H}), 7.31$ (d, $J=8.6 \mathrm{~Hz}, 8 \mathrm{H}), 7.25$ (d, $J$ = $8.6 \mathrm{~Hz}, 32 \mathrm{H}), 5.31(\mathrm{~s}, 8 \mathrm{H}), 5.18(\mathrm{~s}, 8 \mathrm{H}), 4.20(\mathrm{t}, \mathrm{J}=6.4 \mathrm{~Hz}, 24 \mathrm{H}), 1.96(\mathrm{~m}, 24 \mathrm{H}), 1.27(\mathrm{~m}$, $144 \mathrm{H}), 0.88$ (t, $J=7.0 \mathrm{~Hz}),-2.60$ (s, 10H, NH); ; ${ }^{13} \mathrm{C}$ NMR (100 MHz, $\left.\mathrm{CDCl}_{3}\right) \delta 159.0,158.5$, 135.6, 135.1, 134.4, 128.6, 127.9, 113.0, 112.7, 70.1, 68.3, 68.1, 31.9, 29.6, 29.5, 29.4, 26.2, 25.7, 22.7, 14.1; UV/Vis ( $\lambda_{\max }$, nm, dichloromethane) 422.1, 518.6, 555.3, 593.1, 650.4; MALDI TOF $6496\left(\mathrm{MH}^{+}\right)$.

5, 10, 15, 20-Tetrakis(4-(4-(4-(5,10,15-tris(4-(3,5-(3,5-bis(benzyloxy)benzyloxy)benzyloxy)phenyl)porphyrin-20-yl)fenoxymethyl)benzyloxy)phenyl)porphyrin (7c). Pentaporphyrin 7c was prepared from 6c $(50 \mu \mathrm{mol})$ and $\mathrm{Zn}[1]$ (10 $\mu \mathrm{mol})$ in $40 \%$ yield. The reaction was carried out in dimethylformamide with potassium carbonate base $(50 \mu \mathrm{mol})$ at $50^{\circ} \mathrm{C}$.

The NMR spectra were broadened, GPC $\mathrm{M}_{\mathrm{n}}=11305$, PD = 1.04; MALDI TOF $12588\left(\mathrm{MH}^{+}\right)$

\section{Acknowledgements}

We thank the Ministerie voor Wetenschapsbeleid, the FWO-Vlaanderen and the Katholieke Universiteit Leuven for their financial support. S. S. thanks the I. W. T. for a predoctoral fellowship.

\section{References}

1. Jin, R.-H.; Aida, T.; Inoue, S. J. Chem. Soc., Chem. Commun. 1993, 1260.

2. Dandliker, P.J. ; Diederich, F. ; Gross, M. ; Knobler, C. B.; Louati, A.; Sanford, E.M.; Angew. Chem. Int. Ed. Engl. 1994, 33,1739.

3. (a) Dandliker, P.J. ; Diederich, F. ; Gisselbrecht, P.-J.; Louati,A.; Gross, M. Angew. Chem. Int. Ed. Engl. 1996, 34, 2725 (b)Weyermann, P, Diederich, F. Helv. Chim. Acta 2002, 85, 599.

4. Bhyrappa, P.; Young, J. K.; Moore, J. S., Suslick, K. S. J. Am. Chem. Soc. 1996, 118, 5708. 
5. Pollak, K.W.; Leon, J.W.; Fréchet, J.M.J.; Maskus, M.; Abruna, H. D. Chem. Mater. 1998, 10, 30.

6. (a) Sadamoto, R.; Tomioka, N.; Aida, T. J. Am. Chem. Soc. 1996, 118, 3978. (b) Tomoyose, Y.; Jiang, D.L. ; Jin, R.H. ; Aida, T.; Yamashita, T. ; Horic, K. Macromolecules 1996, 29, 5236.

7. (a) Jiang, D.L.; Aida, T. J. Am. Chem. Soc. 1998, 120, 10895 (b) Kimura, M.; Shiba, T.; Muto, T.; Shirai, K.; Hanabusa, K. Macromolecules 1999, 32, 8237.

8. Choi, M. S.; Aida, T.; Yamazaki, T. Yamazaki, I.; Chem. Eur. J. 2002, 8, 2668.

9. Norsten, T.; Branda, N. J. Chem. Soc., Chem. Commun. 1998, 1257.

10. Capitosti, G.J.; Cramer, S.J.; Rajesh, C.S.; Modarelli, D.A. Org. Lett. 2001, 3, 1645.

11. Vinogradov, S.A.; Wilson, D. F. Chem. Eur. J. 2000, 6, 2456.

12. Matos, M.S.; Hofkens, J. Verheijen, W.; De Schryver, F.C.; Hecht, S.; Pollak, K. W.; Fréchet, J.M.J.; Forier, B.; Dehaen, W. Macromolecules 2000, 33, 2967.

13. Review on multiporphyrin systems : Burrell, A.K.; Officer, D.L.; Pliege, P.G.; Reid, D.C.W. Chem. Rev. 2001, 101, 2751.

14. L’abbé, G.; Forier, B; Dehaen,W. J. Chem. Soc., Chem. Commun. 1996, 2143.

15. Forier, B. ; Dehaen, W. Tetrahedron 1999, 55, 9829.

16. Hawker, C.J. ; Fréchet, J.M.J. J. Am. Chem. Soc. 1990, 112, 7638.

17. Hofkens J.; Latterini L.; Vanoppen P.; Faes H.; Jeuris K.; De Feyter S.; Kerimo J.; Barbara P.F.; DeSchryver F.C.; Rowan A.E., Nolte R.J.M. J. Phys. Chem. B 1999, 101, 10588. 